\title{
Analysis of risk factors for pulmonary infection in patients with minimally invasive esophagectomy
}

\author{
GUANGYUAN LIU ${ }^{1}$, LIN PENG $^{1}$, BIN LIU $^{2}$, KANGNING WANG $^{1}$ and YONGTAO HAN ${ }^{1}$ \\ ${ }^{1}$ Ward 1, Department of Thoracic Surgery and ${ }^{2}$ Clinical Research Standardized Treatment Ward, \\ Department of Medical Oncology, Sichuan Cancer Hospital and Institute, Chengdu, Sichuan 610041, P.R. China
}

Received September 21, 2018; Accepted January 24, 2019

DOI: $10.3892 / \mathrm{ol} .2019 .9987$

\begin{abstract}
Clinical manifestations of patients with pulmonary infection after minimally invasive esophagectomy were analyzed. A total of 500 patients undergoing minimally invasive esophagectomy in Sichuan Cancer Hospital and Institute from January 2015 to December 2016 were consecutively selected, among which 124 patients with pulmonary infection after surgery were taken as the infection group, and the remaining 376 patients were taken as the control group. The clinical data of all patients were collected. The pulmonary infection rate after minimally invasive esophagectomy was calculated, and clinical factors with difference were analyzed using the multivariate logistic regression analysis. There were significant differences in age, long-term smoking history, presence or absence of concurrent basic diseases and vital capacity $(\mathrm{P}<0.01)$. The application time of antibiotics and antacids and hospitalization duration in the infection group were obviously longer than those in the control group $(\mathrm{P}<0.01)$. Age, long-term smoking history, vital capacity, application time of antibiotics and antacids, hospitalization duration and concurrent diabetes mellitus/coronary heart disease/diseases of respiratory system increase the risk of postoperative pulmonary infection in patients with esophageal cancer, and age, long-term smoking history, diabetes mellitus, diseases of respiratory system and hospitalization duration are independent risk factors for pulmonary infection in patients after minimally invasive esophagectomy.
\end{abstract}

\section{Introduction}

Esophageal cancer refers to the malignant tumor of epithelial tissues derived from the esophagus. The number of deaths

Correspondence to: Dr Yongtao Han, Ward 1, Department of Thoracic Surgery, Sichuan Cancer Hospital and Institute, 55, Section 4, Renmin South Road, Chengdu, Sichuan 610041, P.R. China

E-mail: h343fv@163.com

Key words: minimally invasive esophagectomy, pulmonary infection, risk factors due to esophageal cancer is approximately 500,000 every year worldwide (1). The pathogenesis of esophageal cancer is related to a variety of factors, such as chemical factors (nitrosamine), biological factors (fungus), deficiency of vitamins and trace elements and dietary habits. People with different genetic backgrounds have different susceptibility to esophageal cancer $(2,3)$. Therefore, the epidemiological characteristics of esophageal cancer have certain regional features. The incidence and mortality rate of esophageal cancer vary from country to country, and its distribution in population is related to age, sex, occupation and race (4). The symptoms occur with varying degrees, and are slow to develop, and most patients are diagnosed at the advanced stage, with poor prognosis and a 5-year survival rate of up to $20 \%(5,6)$.

Currently, surgical resection is a preferred therapeutic method for esophageal cancer when the patient's physical capacity allows (7). However, even after resection, the prognosis and quality of life of patients will be severely affected by complications, metastasis, recurrence and progression of esophageal cancer. The 5-year survival rate is only $\sim 25 \%(8,9)$. As a new surgical method, minimally invasive esophagectomy is performed for patients through thoracoscope and laparoscope, which reduces the patient's trauma, alleviates the patient's pain and accelerates the recovery of the patient compared with conventional thoracotomy (10). However, pulmonary infection is still the first postoperative complication, as well as one of the main causes of postoperative death except the quality of surgery itself, seriously harming surgical quality and prognosis of patients (11). In the present study, relative factors to pulmonary infection in patients after minimally invasive esophagectomy were analyzed, so as to provide a theoretical basis for the prevention and treatment of pulmonary infection after radical surgery for esophageal cancer, and reduce the incidence and mortality rate of postoperative complications.

\section{Patients and methods}

Subjects of study and grouping. General clinical data of 500 patients undergoing minimally invasive esophagectomy in Sichuan Cancer Hospital and Institute (Chengdu, China) from January 2015 to December 2016 were collected, and patients were grouped, among which 124 patients with pulmonary infection after surgery were taken as the infection group with 
Table I. Comparison of the basic clinical data between the two groups of patients.

\begin{tabular}{|c|c|c|c|c|}
\hline Factors & Infection group $(\mathrm{n}=124)$ & Control group $(\mathrm{n}=376)$ & $\chi^{2 / t}$ & P-value \\
\hline Age (years) & & & 55.47 & 0.001 \\
\hline$\geq 60$ & $84(67.74)$ & $113(30.05)$ & & \\
\hline$<60$ & $40(32.26)$ & $263(69.05)$ & & \\
\hline $\operatorname{Sex}[\mathrm{n}(\%)]$ & & & 0.22 & 0.64 \\
\hline Male & 89 (71.77) & $278(73.94)$ & & \\
\hline Female & $35(28.23)$ & $98(26.06)$ & & \\
\hline BMI $\left(\mathrm{kg} / \mathrm{m}^{2}\right)$ & $24.11 \pm 5.86$ & $25.32 \pm 6.32$ & 1.95 & 0.06 \\
\hline Marital status [n (\%)] & & & 0.66 & 0.72 \\
\hline Single & $5(4.03)$ & $16(4.26)$ & & \\
\hline Married & $80(64.52)$ & $256(68.09)$ & & \\
\hline Divorced & $39(31.45)$ & $104(27.66)$ & & \\
\hline Educational level & & & 2.20 & 0.33 \\
\hline Junior high school and below & $42(33.87)$ & $125(33.24)$ & & \\
\hline Senior high school and junior college & $54(43.55)$ & $142(37.77)$ & & \\
\hline Undergraduate and above & $28(22.58)$ & 109 (28.99) & & \\
\hline Long-term smoking history [n (\%)] & & & 49.98 & 0.001 \\
\hline Yes & $66(53.23)$ & $76(20.21)$ & & \\
\hline No & $58(46.77)$ & $300(79.79)$ & & \\
\hline Drinking history [n (\%)] & & & 0.00 & 0.96 \\
\hline Yes & $37(29.84)$ & $113(30.05)$ & & \\
\hline No & $87(70.16)$ & $263(69.95)$ & & \\
\hline Basic diseases [n (\%)] & & & 12.39 & 0.001 \\
\hline Yes & $78(62.90)$ & $168(44.68)$ & & \\
\hline No & $46(37.10)$ & $208(55.32)$ & & \\
\hline Chemoradiotherapy [n (\%)] & & & 0.04 & 0.84 \\
\hline Yes & $111(89.52)$ & $339(90.16)$ & & \\
\hline No & $13(10.48)$ & $37(9.84)$ & & \\
\hline Vital capacity (ml) & $870.12 \pm 139.22$ & $1287.31 \pm 110.34$ & 30.37 & 0.001 \\
\hline \multicolumn{5}{|l|}{ Pathological type } \\
\hline I-II & $108(87.10)$ & $338(89.89)$ & 0.76 & 0.38 \\
\hline III-IV & $16(12.90)$ & $38(10.11)$ & & \\
\hline
\end{tabular}

an incidence rate of pulmonary infection of $24.8 \%$, and the remaining 376 patients without pulmonary infection were taken as the control group. Among the 500 patients, there were 367 males and 133 females aged $60.13 \pm 10.83$ years, and 60 years was the cut-off value of the patient's age. The vital capacity was $1101.83 \pm 124.73 \mathrm{ml}$, and $1100 \mathrm{ml}$ was the cut-off value of the patients' vital capacity. The inclusion criteria were the following: i) patients meeting the diagnostic criteria for esophageal cancer (12); ii) patients receiving esophageal cancer staging according to the UICC-AJCC TNM staging criteria (13); iii) patients undergoing minimally invasive esophagectomy; iv) patients in the infection group meeting the diagnostic criteria for pulmonary infection (14); and v) patients who had signed the informed consent and willing to cooperate in the study. The exclusion criteria were the following: i) patients with incomplete clinical data; ii) patients complicated with other serious basic organ diseases in the liver or kidney; iii) patients complicated with mental disease or mental disorder; or v) patients with primary immune system diseases and immunocompromised.

The present study was approved by the Ethics Committee of Sichuan Cancer Hospital and Institute.

Method. After clinical data of all patients were collected, whether there were significant differences in the sex, age, vital capacity, history of disease and operation time were compared and analyzed between the two groups of patients. The pulmonary infection rate after minimally invasive esophagectomy was calculated, and the clinical factors with difference were analyzed using the multivariate logistic regression analysis.

Statistical analysis. Statistical Product and Service Solutions (SPSS) 19.0 software package (IBM Corp., Armonk, NY, USA) was used for data analysis and processing. Measurement data were expressed as mean \pm SD and significant differences were compared using t-test. Enumeration data were 
Table II. Comparison of type and proportion of main diseases in basic diseases.

\begin{tabular}{|c|c|c|c|c|}
\hline Factors & Infection group $(n=124)$ & Control group $(\mathrm{n}=376)$ & $\chi^{2}$ & P-value \\
\hline Hypertension & & & 0.00 & 0.94 \\
\hline Yes & $49(39.52)$ & $150(39.89)$ & & \\
\hline No & $75(60.48)$ & $226(60.11)$ & & \\
\hline Coronary heart disease & & & 5.32 & 0.001 \\
\hline Yes & $20(16.13)$ & $33(8.78)$ & & \\
\hline No & $104(83.87)$ & $343(91.22)$ & & \\
\hline Diabetes mellitus & & & 13.37 & 0.001 \\
\hline Yes & $25(20.16)$ & $37(9.84)$ & & \\
\hline No & $99(79.84)$ & $339(90.16)$ & & \\
\hline Diseases of respiratory system & & & 38.40 & 0.001 \\
\hline Yes & $37(29.84)$ & $30(7.98)$ & & \\
\hline No & $87(70.16)$ & $346(92.02)$ & & \\
\hline
\end{tabular}

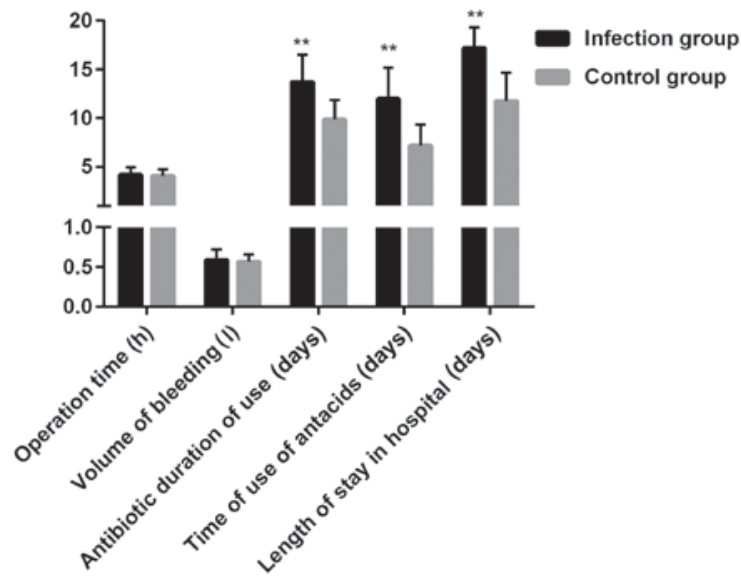

Figure 1. Comparison of treatment conditions between the two groups of patients. The operation time and amount of intraoperative bleeding were not significantly different between the two groups $(\mathrm{P}>0.05)$, but the application time of antibiotics and antacids and hospitalization duration in the infection group were obviously longer than those in the control group. ${ }^{* *} \mathrm{P}<0.01$.

analyzed using the Chi-square test. After univariate analysis, multivariate logistic regression analysis was performed, and factors $(\mathrm{P}<0.05)$ that were independent risk factors for pulmonary infection in patients after minimally invasive esophagectomy. The significance level was set as $\alpha=0.05$.

\section{Results}

Comparison of basic clinical data between the two groups of patients. There were no differences in sex, body mass index (BMI), marital status, educational level, drinking history, chemoradiotherapy and pathological type between the two groups $(\mathrm{P}>0.05)$, but there were significant differences in age, long-term smoking history, presence or absence of concurrent basic diseases and vital capacity $(\mathrm{P}<0.001)$. The age in the infection group was significantly higher than that in the control group, the number of patients with long-term smoking history and basic diseases in the infection group were significantly larger than that in the control group, and the vital capacity in the infection group was significantly lower than that in the control group (Table I). The main concurrent basic diseases in both groups included hypertension, coronary heart disease, diabetes mellitus and diseases of respiratory system. There was no difference in the proportion of patients complicated with hypertension between the groups $(\mathrm{P}>0.05)$, but there were differences in the proportion of patients complicated with coronary heart disease, diabetes mellitus and diseases of respiratory system $(\mathrm{P}<0.001)$ (Table II).

Comparison of treatment conditions between the two groups of patients. The operation time was $4.21 \pm 0.75 \mathrm{~h}$ in the infection group and $4.09 \pm 0.66 \mathrm{~h}$ in the control group, the amount of intraoperative bleeding was $0.59 \pm 0.13 \mathrm{ml}$ in the infection group and $0.57 \pm 0.09 \mathrm{ml}$ in the control group, the application time of antibiotics was $13.72 \pm 2.76$ days in the infection group and $9.87 \pm 1.98$ days in the control group, the application time of antacids was $11.98 \pm 3.21$ days in the infection group and $7.21 \pm 2.11$ days in the control group, and the hospitalization duration was $17.21 \pm 2.11$ days in the infection group and $11.76 \pm 2.92$ days in the control group. The operation time and amount of intraoperative bleeding were not significantly different between the two groups $(\mathrm{P}>0.05)$, but the application time of antibiotics and antacids and hospitalization duration in the infection group were obviously longer than those in the control group $(\mathrm{P}<0.05)$ (Fig. 1).

Comparison of infection rate under difference factors. The incidence rate of pulmonary infection in 500 patients after minimally invasive esophagectomy was $24.80 \%$. According to the stratification analysis based on difference factors, it was found that the pulmonary infection rate after minimally invasive esophagectomy was obviously higher in patients aged $\geq 60$ years, with a long-term smoking history and vital capacity $<1100 \mathrm{ml}$, and complicated with coronary heart disease/diabetes mellitus/diseases of respiratory system than that in patients aged $<60$ years, without a long-term smoking history, with vital capacity $\geq 1100 \mathrm{ml}$, and without coronary 
Table III. Comparison of postoperative pulmonary infection rate under difference factors.

\begin{tabular}{|c|c|c|c|c|c|}
\hline Difference factor & Total case $(n=500)$ & Case of infection $(n=124)$ & Infection rate $(\%)$ & $\chi^{2}$ & P-value \\
\hline Age (years) & & & & 55.47 & 0.001 \\
\hline$\geq 60$ & 197 & 84 & 42.64 & & \\
\hline$<60$ & 303 & 40 & 13.20 & & \\
\hline Long-term smoking history & & & & 49.98 & 0.001 \\
\hline Yes & 142 & 66 & 46.48 & & \\
\hline No & 358 & 58 & 16.20 & & \\
\hline Vital capacity (ml) & & & & 24.09 & 0.001 \\
\hline$\geq 1100$ & 212 & 76 & 16.67 & & \\
\hline$<1100$ & 288 & 48 & 35.85 & & \\
\hline Coronary heart disease & & & & 5.319 & 0.02 \\
\hline Yes & 53 & 20 & 37.74 & & \\
\hline No & 447 & 104 & 23.27 & & \\
\hline Diabetes mellitus & & & & 9.14 & 0.02 \\
\hline Yes & 62 & 25 & 40.32 & & \\
\hline No & 438 & 99 & 22.60 & & \\
\hline Diseases of respiratory system & & & & 38.40 & 0.001 \\
\hline Yes & 67 & 37 & 55.22 & & \\
\hline No & 433 & 87 & 20.09 & & \\
\hline
\end{tabular}

Table IV. Multivariate logistic regression analysis.

\begin{tabular}{lccccc}
\hline Difference factor & B & Wald & OR & $95 \%$ CI & P-value \\
\hline Age & 0.19 & 5.204 & 1.28 & $1.08-1.56$ & 0.02 \\
Long-term smoking history & 0.62 & 15.41 & 11.80 & $8.83-24.32$ & 0.001 \\
Diabetes mellitus & 0.42 & 1.43 & 1.32 & $0.32-4.32$ & 0.13 \\
Coronary heart disease & 0.12 & 2.98 & 3.46 & $1.76-9.533$ & 0.07 \\
Diabetes mellitus & 0.24 & 7.45 & 4.78 & $1.33-7.43$ & 0.01 \\
Diseases of respiratory system & 1.23 & 16.56 & 5.53 & $2.43-11.34$ & 0.001 \\
Application time of antibiotics & 0.32 & 2.21 & 3.42 & $1.43-7.43$ & 0.22 \\
Application time of antacids & 0.91 & 2.54 & 4.32 & $0.98-8.54$ & 0.49 \\
Hospitalization duration & 1.72 & 9.23 & 3.52 & $1.21-7.43$ & 0.001 \\
\hline
\end{tabular}

OR, odds ratio; CI, confidence interval.

heart disease/diabetes mellitus/diseases of respiratory system $(\mathrm{P}<0.05)$ (Table III).

Multivariate logistic regression analysis of difference factor. Multivariate logistic regression analysis was performed for difference factor screened with pulmonary infection after minimally invasive esophagectomy as the dependent variable, and results demonstrated that age $(\mathrm{OR}=1.28, \mathrm{P}=0.02)$, long-term smoking history $(\mathrm{OR}=11.80, \mathrm{P}<0.001)$, diabetes mellitus $(\mathrm{OR}=4.78, \mathrm{P}=0.01)$, diseases of respiratory system $(\mathrm{OR}=5.53, \mathrm{P}<0.001)$ and hospitalization duration $(\mathrm{OR}=3.52$, $\mathrm{P}<0.001)$ were independent risk factors for pulmonary infection in patients after minimally invasive esophagectomy (Table IV).

\section{Discussion}

The incidence rate of esophageal cancer, a common malignant tumor in the digestive system, is among the top 10 in the world, which is second only to gastric cancer in China. The number of patients with esophageal cancer in China accounts for half of the total worldwide. The elderly patients are in the majority, and its incidence rate in male is about three times that in female $(15,16)$. The prognosis of esophageal cancer is poor, and its mortality rate is high in China. The preferred therapeutic method is surgical resection supplemented by chemoradiotherapy (17). Pulmonary infection is one of the major complications of minimally invasive esophagectomy (18). To reduce the incidence rate of complications 
after surgery for esophageal cancer and the mortality rate of patients, relative factors to pulmonary infection after minimally invasive esophagectomy were investigated in the present study, so as to provide a theoretical basis for the prevention and treatment of pulmonary infection after radical surgery for esophageal cancer.

Patients in the infection group had pulmonary infection after surgery for esophageal cancer, but no pulmonary infection occurred in the control group after surgery. There were no differences in the sex, BMI, marital status and educational level between the two groups of patients, but there were significant differences in the age, vital capacity, long-term smoking history and presence or absence of concurrent basic diseases. When stratifying the infection rate for these different factors, we found that the high age, long-term smoking history and concurrent diabetes mellitus, coronary heart disease and diseases of respiratory system may increase the probability of pulmonary infection in patients after minimally invasive esophagectomy. The comparison of treatment factors between the two groups of patients revealed that there were no significant differences in the operation time and amount of intraoperative bleeding between the two groups, and the application time of antibiotics and antacids and hospitalization duration in the infection group were significantly longer than those in the control group, suggesting that the application time of antibiotics and antacids and hospitalization duration may affect the incidence rate of pulmonary infection in patients after minimally invasive esophagectomy. It was found in the multivariate logistic regression analysis that the age, long-term smoking history, diabetes mellitus, diseases of respiratory system and hospitalization duration were independent risk factors for pulmonary infection in patients after minimally invasive esophagectomy. Wang et al (10) studied the risk factors for pneumonia in patients with esophageal cancer after transthoracic esophagectomy and found that BMI, age and concurrent diabetes mellitus were major influencing factors. In our study, however, BMI did not affect the incidence rate of postoperative pulmonary infection in patients with esophageal cancer. The possible reason is that open surgery was adopted in the study of Wang et al (10), while the minimally-invasive surgery was adopted in this study. Wang et al (10) studied the relative factors to pulmonary infection after surgery for esophageal cancer and found that the age, operation time, other concurrent basic diseases and recurrent laryngeal nerve injury are risk factors for postoperative infection, which, except the operation time, are consistent with results in the present study. The possible reason is that the study of Wang et al (10) was a comprehensive study containing various surgical methods, while only risk factors for minimally invasive esophagectomy were explored in this study, thus, leading to different results. According to the study of Saito et al (19), the cellular immunodeficiency after surgery for esophageal cancer can promote the occurrence of infection. Besides, Saito et al (20) found that the increase in superoxide anion production (SOP) of polymorphonuclear neutrophil can predict the postoperative infection of esophageal cancer. Therefore, some indexes reflecting cellular immunodeficiency, such as T cells, B cells and phytohaemagglutinin (PHA)-induced transformation, and SOP can be added to future research, so as to investigate the postoperative cellular immunodeficiency and SOP in patients after minimally invasive esophagectomy and their correlations with pulmonary infection.

It was found in the comparison of the type and proportion of basic diseases between the two groups that the proportions of coronary heart disease and diabetes mellitus were not high in either group (only approximately 20-30 cases), which may affect the results of the analysis. Therefore, the sample size needs to be increased for repeated verification. Moreover, several basic diseases could occur simultaneously in patients, such as hypertension and diabetes mellitus. To improve the efficiency, however, such a situation was not considered in the analysis of the type and proportion of basic diseases in this study, but the focus was placed on one variable. Multiple variables were considered in the multivariate logistic regression analysis, and it was found that the history of diabetes mellitus and diseases of respiratory system were independent risk factors for pulmonary infection in patients after minimally invasive esophagectomy. Thus, the history of diabetes mellitus and/or diseases of respiratory system increase the risk of pulmonary infection after minimally invasive esophagectomy, regardless of the type and number of other concurrent diseases. However, whether the rate of pulmonary infection after minimally invasive esophagectomy is associated with the types and numbers of concurrent several basic diseases, needs larger-sample studies for verification.

Due to the limitations of the retrospective study, we did not collect all the immune indicators data of patients to judge the immune function, and the judgement of immune function is complex. At present, we need to combine multiple indicators and make a comprehensive judgement (21). Also, the factors affecting immunity are also complex and varied (22). In this study, all the subjects were middle-aged and elderly individuals, with an average age of $60.13 \pm 10.83$ years, and their immunity gradually declined with age. In addition, all the subjects were suffering from esophageal cancer, and esophageal cancer itself can affect the immunity of patients (23). Therefore, considering that patients' own immunity may affect the probability of pulmonary infection after minimally invasive surgery (24), we excluded patients with primary immune system diseases and those immunocompromised. For those with secondary immune dysfunction or immunocompromise, we summarized the effect of secondary immunity on pulmonary infection after surgery into their primary diseases, such as diabetes mellitus patients with secondary immunocompromise, which affects the probability of pulmonary infection after surgery (25). This effect is thought to be caused by diabetes, not by low immunity, because low immunity here is only a secondary effect of diabetes. Since we excluded the patients with primary immune dysfunction or immunocompromise, the age of the two groups was not different, and minimally invasive esophagectomy was also performed. Thus, although immune dysfunction or immunocompromise may have an impact on the incidence of postoperative pulmonary infection, it is not the main factor in the present study.

In conclusion, age, long-term smoking history, vital capacity, application time of antibiotics and antacids, hospitalization duration and concurrent diabetes mellitus/coronary heart disease/diseases of respiratory system increase the risk of postoperative pulmonary infection in patients with esophageal cancer, and age, long-term smoking history, diabetes mellitus, 
diseases of respiratory system and hospitalization duration are independent risk factors for pulmonary infection in patients after minimally invasive esophagectomy.

\section{Acknowledgements}

Not applicable.

\section{Funding}

No funding was received.

\section{Availability of data and materials}

The datasets used and/or analyzed during the present study are available from the corresponding author on reasonable request.

\section{Authors' contributions}

GL drafted the manuscript. GL and LP were mainly involved in collecting and interpreting the general data of patients. BL and KW analyzed the pulmonary infection. GL, BL and YH were responsible for the analysis of clinical factors. All authors read and approved the final manuscript.

\section{Ethics approval and consent to participate}

The present study was approved by the Ethics Committee of Sichuan Cancer Hospital and Institute (Chengdu, China). Signed informed consents were obtained from the patients and/or guardians.

\section{Patient consent for publication}

Not applicable.

\section{Competing interests}

The authors declare that they have no competing interests.

\section{References}

1. Zhao L, Ge J, Li W, Luo Y and Chai Y: Minimally invasive esophageal resection and intrathoracic anastomosis for lower thoracic esophageal cancer with single position. J Thorac Dis 7: $1486-1488,2015$.

2. Farran L, Llop J, Sans M, Kreisler E, Miró M, Galan M and Rafecas A: Efficacy of enteral decontamination in the prevention of anastomotic dehiscence and pulmonary infection in esophagogastric surgery. Dis Esophagus 21: 159-164, 2008.

3. Xie FJ, Zhang YP, Zheng QQ, Jin HC, Wang FL, Chen M, Shao L, Zou DH, Yu XM and Mao WM: Helicobacter pylori infection and esophageal cancer risk: An updated meta-analysis. World J Gastroenterol 19: 6098-6107, 2013.

4. Berry MF, Atkins BZ, Tong BC, Harpole DH, D'Amico TA and Onaitis MW: A comprehensive evaluation for aspiration after esophagectomy reduces the incidence of postoperative pneumonia. J Thorac Cardiovasc Surg 140: 1266-1271, 2010.

5. Tachimori Y, Ozawa S, Numasaki H, Fujishiro M, Matsubara H, Oyama T, Shinoda M, Toh Y, Udagawa $\mathrm{H}$ and Uno T; Registration Committee for Esophageal Cancer of the Japan Esophageal Society: Comprehensive Registry of Esophageal Cancer in Japan, 2009. Esophagus 13: 110-137, 2016.

6. Grade M, Beham AW, Schüler P, Kneist W and Ghadimi BM Pelvic intraoperative neuromonitoring during robotic-assisted low anterior resection for rectal cancer. J Robot Surg 10: 157-160, 2016.
7. Su H, Lin F, Deng X, Shen L, Fang Y, Fei Z, Zhao L, Zhang X, Pan H, Xie D, et al: Profiling and bioinformatics analyses reveal differential circular RNA expression in radioresistant esophageal cancer cells. J Transl Med 14: 225, 2016.

8. Ma Q, Liu W, Jia R, Jiang F, Duan H, Lin P, Zhang L, Long H, Zhao $\mathrm{H}$ and $\mathrm{Ma} \mathrm{G}$ : Inflammation-based prognostic system predicts postoperative survival of esophageal carcinoma patients with normal preoperative serum carcinoembryonic antigen and squamous cell carcinoma antigen levels. World J Surg Oncol 14: $141,2016$.

9. Raymond DP, Seder CW, Wright CD, Magee MJ, Kosinski AS, Cassivi SD, Grogan EL, Blackmon SH, Allen MS, Park BJ, et al: Predictors of major morbidity or mortality after resection for esophageal cancer: A Society of Thoracic Surgeons General Thoracic Surgery Database Risk Adjustment Model. Ann Thorac Surg 102: 207-214, 2016.

10. Wang LJ, Gu LB, Jiang DM, Gao R, Xu ZP, Wan MF and Lu Z: Ridge regression analysis of pulmonary infection rate after transthoracic esophagectomy for esophageal cancer. Zhonghua Yi Xue Za Zhi 92: 1310-1313, 2012 (In Chinese).

11. Yamamoto M, Weber JM, Karl RC and Meredith KL: Minimally invasive surgery for esophageal cancer: Review of the literature and institutional experience. Cancer Contr 20: 130-137, 2013.

12. Meves V, Behrens A and Pohl J: Diagnostics and early diagnosis of esophageal cancer. Viszeralmedizin 31: 315-318, 2015.

13. Talsma K, van Hagen P, Grotenhuis BA, Steyerberg EW, Tilanus HW, van Lanschot JJ and Wijnhoven BP: Comparison of the 6th and 7th Editions of the UICC-AJCC TNM Classification for Esophageal Cancer. Ann Surg Oncol 19: 2142-2148, 2012.

14. Rogers ML, Taylor R and Beggs FD: Antibiotic prophylaxis in general thoracic surgery in the UK. Eur J Cardiothorac Surg 18: 375-376, 2000.

15. Ida S, Watanabe M, Yoshida N, Baba Y, Umezaki N, Harada K, Karashima R, Imamura Y, Iwagami S and Baba $\mathrm{H}$ : Sarcopenia is a predictor of postoperative respiratory complications in patients with esophageal cancer. Ann Surg Oncol 22: 4432-4437, 2015.

16. Li J, Zhang BZ, Qin YR, Bi J, Liu HB, Li Y, Cai MY, Ma S, Chan KW, Xie D, et al: CD68 and interleukin 13, prospective immune markers for esophageal squamous cell carcinoma prognosis prediction. Oncotarget 7: 15525-15538, 2016.

17. Wang G, Chen H, Liu J, Ma Y and Jia H: A comparison of postoperative early enteral nutrition with delayed enteral nutrition in patients with esophageal cancer. Nutrients 7: 4308-4317, 2015.

18. Peyre CG and Peters JH: Minimally invasive surgery for esophageal cancer. Surg Oncol Clin N Am 22: 15-25, 2013.

19. Saito T, Shimoda K, Shigemitsu Y, Kinoshita T, Kuwahara A, Miyahara $\mathrm{M}$ and Kobayashi M: Complications of infection and immunologic status after surgery for patients with esophageal cancer. J Surg Oncol 48: 21-27, 1991

20. Saito T, Shigemitsu Y, Kinoshita T, Katsuta T, Shimoda K, Miyahara M and Kobayashi M: Impaired neutrophil bactericidal activity correlates with the infection occurring after surgery for esophageal cancer. J Surg Oncol 51: 159-163, 1992.

21. Piccolo V, Baroni A, Russo T and Schwartz RA: Ruocco's immunocompromised cutaneous district. Int J Dermatol 55: 135-141, 2016.

22. Qiu Y, Zhao R, Yun MM, Han X, Yun F, Liu B, Zhou E, Ouyang X and Yun S: Immunity enhancement in immunocompromised gastrointestinal cancer patients with allogeneic umbilical cord blood mononuclear cell transfusion. Biomed Res Int 2017: 5945190, 2017.

23. Kitada M, Matsuda Y, Hayashi S, Ishibashi K, Oikawa K and Miyokawa N: Esophageal schwannoma: A case report. World J Surg Oncol 11: 253, 2013.

24. Ozcan HN, Gormez A, Ozsurekci Y, Karakaya J, Oguz B, Unal S, Cetin M, Ceyhan M and Haliloglu M: Magnetic resonance imaging of pulmonary infection in immunocompromised children: Comparison with multidetector computed tomography. Pediatr Radiol 47: 146-153, 2017.

25. Fernández-Real JM and Pickup JC: Innate immunity, insulin resistance and type 2 diabetes. Diabetologia 55: 273-278, 2012.

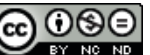

This work is licensed under a Creative Commons Attribution-NonCommercial-NoDerivatives 4.0 International (CC BY-NC-ND 4.0) License. 